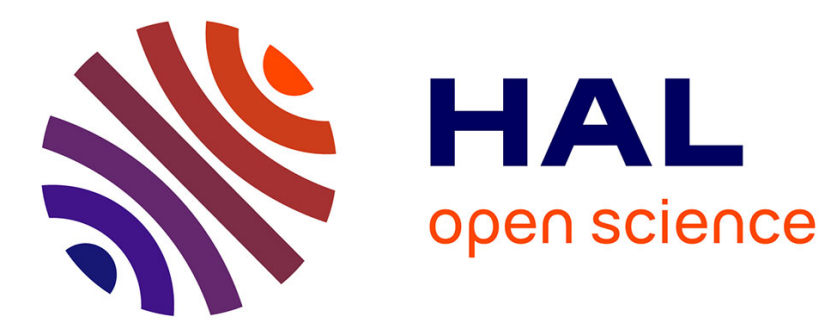

\title{
Personnel et internés dans les camps français de la guerre d'Algérie
}

\author{
Sylvie Thénault
}

\section{To cite this version:}

Sylvie Thénault. Personnel et internés dans les camps français de la guerre d'Algérie. Politix, 2005, n 69 (1), pp.63. 10.3917/pox.069.0063 . hal-02355819

\section{HAL Id: hal-02355819 \\ https://hal.science/hal-02355819}

Submitted on 12 Nov 2019

HAL is a multi-disciplinary open access archive for the deposit and dissemination of scientific research documents, whether they are published or not. The documents may come from teaching and research institutions in France or abroad, or from public or private research centers.
L'archive ouverte pluridisciplinaire HAL, est destinée au dépôt et à la diffusion de documents scientifiques de niveau recherche, publiés ou non, émanant des établissements d'enseignement et de recherche français ou étrangers, des laboratoires publics ou privés. 


\section{PERSONNEL ET INTERNÉS DANS LES CAMPS FRANÇAIS DE LA GUERRE D'ALGÉRIE}

Entre stéréotypes coloniaux et combat pour l'indépendance

\section{Sylvie Thénault}

De Boeck Supérieur | « Politix »

2005/1 nº 69 | pages 63 à 81

ISSN 0295-2319

ISBN 9782200920661

Article disponible en ligne à l'adresse :

https://www.cairn.info/revue-politix-2005-1-page-63.htm

Distribution électronique Cairn.info pour De Boeck Supérieur.

(C) De Boeck Supérieur. Tous droits réservés pour tous pays.

La reproduction ou représentation de cet article, notamment par photocopie, n'est autorisée que dans les limites des conditions générales d'utilisation du site ou, le cas échéant, des conditions générales de la licence souscrite par votre établissement. Toute autre reproduction ou représentation, en tout ou partie, sous quelque forme et de quelque manière que ce soit, est interdite sauf accord préalable et écrit de l'éditeur, en dehors des cas prévus par la législation en vigueur en France. Il est précisé que son stockage dans une base de données est également interdit. 


\section{Personnel et internés dans les camps français de la guerre d'Algérie Entre stéréotypes coloniaux et combat pour l'indépendance}

Sylvie ThÉNAULT

Résumé - Cet article s'intéresse aux camps français de la guerre d'Algérie. En métropole comme en Algérie, ces camps ont été gérés avec un pragmatisme conduisant à la limitation du personnel embauché et recruté pour la direction, l'encadrement et la surveillance des internés. Seuls les soldats moniteurs d'action psychologique en Algérie et les civils chargés de l'action sociale dans les camps métropolitains, pendant l'un de l'autre, ont été choisis sur des critères témoignant de l'intérêt porté par les autorités à la réussite de leur mission : enrayer l'adhésion des internés à la cause du FLN. Cette conception de l'interné comme un être manipulable, cependant, est contredite par les actions de résistance et d'opposition des internés à l'intérieur des camps. En métropole, ils participent ainsi aux combats menés à l'extérieur par le FLN, tandis qu'en Algérie, siège de la guerre, ils sont fragilisés et victimes de traitements dégradants pouvant aller jusqu'aux sévices ou à la mort. 
L es camps d'internement de la guerre d'Algérie restent mal connus ${ }^{1}$. Les travaux historiques consacrés à la pratique « internementale » française ont couvert prioritairement la première et la seconde guerres mondiales, tandis que l'historiographie du conflit franco-algérien n'a pas exploré ce pan de la répression des nationalistes ${ }^{2}$. Depuis une dizaine d'années, en effet, cette historiographie a progressé dans le domaine strictement militaire, grâce à l'exploitation des archives du Service historique de l'armée de terre (SHAT), faisant passer au second plan l'action des autorités civiles et politiques ${ }^{3}$.

Or, celles-ci jouent un rôle essentiel dans la création et la gestion des camps d'internement. Ce sont les gouvernements et les parlementaires qui ont élaboré la législation conduisant à leur ouverture. En métropole, l'administration des camps relève aussi entièrement de la compétence des autorités civiles, ministère de l'Intérieur et préfectures. En Algérie, le Gouvernement général et les préfectures en contrôlent également une partie, ceux désignés par l'euphémisme de "centres d'hébergement», tandis que l'armée gère des centres de détention assimilables, juridiquement, à des lieux d'internement.

Entre 1954 et 1962, la République renoue ainsi avec la «logique d'exception ${ }^{4}$ » qui caractérise son recours à l'internement administratif, voulant qu'à des circonstances exceptionnelles répondent des moyens exceptionnels de répression ; la nature de cette guerre, néanmoins, singularise ces camps de leurs prédécesseurs, dans la mesure où ils sont intégrés au dispositif de la lutte contre l'ennemi. La guerre d'Algérie combine en effet opérations militaires et mesures de maintien de l'ordre, face à un FLN (Front de libération nationale) qui déploie son action en dehors du champ traditionnel de l'affrontement militaire. Les maquis et les cellules terroristes sont soutenus par des réseaux logistiques travaillant à la collecte de fonds, au renseignement, à la propagande, au ravitaillement, à l'hébergement, aux soins des combattants ou militants, etc. À cette panoplie très complète d'activités développées en Algérie, s'ajoute un front métropolitain où le FLN se manifeste essentiellement par ses attentats et sa

1. Seuls quelques articles existent : Bernardot (M.), «Au pays des mille et un camps : approche socio-historique des espaces d'internement en France au XXe siècle", Les cahiers du CERIEM, 10, 2002 ; «Entre répression policière et prise en charge sanitaire et sociale : le cas du centre d'assignation à résidence du Larzac (1957-1963) », Bulletin de l'IHTP, 83, 2004 ; Stora (B.), " La politique des camps d'internement », L’Algérie des Français, Paris, Le Seuil, 1993 ; Thénault (S.), "Assignation à résidence et justice en Algérie ", Le genre humain, 32, 1997 ; "Interner en République : le cas de la France en guerre d'Algérie ", @mnis, 3, 2003 (disponible en ligne : http ://www.univ-brest.fr/amnis/).

2. Farcy (J.-C.), Les camps de concentration de la première guerre mondiale, Paris, Anthropos, 1995 ; Peschanski (D.), La France des camps. L'internement, 1938-1946, Paris, Gallimard, 2002.

3. En témoignent deux grands colloques : Jauffret (J.-C.), Vaïsse (M.), dir., Militaires et guérilla dans la guerre d'Algérie, Bruxelles, Complexe, 2001 ; Jauffret (J.-C.), dir., Des hommes et des femmes en guerre d'Algérie, Paris, Autrement, 2003.

4. D. Peschanski distingue une logique d'exception caractéristique du régime républicain et une logique d'exclusion propre au régime de Vichy (La France des camps, op. cit.). 
pression financière sur les émigrés, véritables bailleurs de fonds du nationalisme. Or, si des opérations militaires sont naturellement opposables à l'ALN (Armée de libération nationale) dans les maquis, elles sont inappropriées à la lutte sur les autres terrains, où la réponse des forces répressives emprunte à l'activité policière de démantèlement de réseaux constitués : arrestation, interrogatoire, détention, condamnation judiciaire lorsque celle-ci peut être obtenue, rétention administrative en l'absence de preuves. C'est dans ce versant policier de la guerre que s'inscrit le recours à la pratique « internementale ». Elle devient une des armes du combat contre les nationalistes.

L'imbrication totale du combat stricto sensu et des opérations de police, cependant, ne se réalise qu'en Algérie, où les militaires obtiennent délégation des pouvoirs d'arrestation, d'interrogatoire et de détention, qu'ils pratiquent en même temps que les autres forces de l'ordre. Alors qu'en métropole, la police et la gendarmerie se chargent seules de la lutte contre le nationalisme, l'intervention des soldats dans le domaine du maintien de l'ordre, auquel se rattache l'internement administratif, marque le territoire algérien.

En quoi cette configuration extérieure aux camps les imprègne-t-elle ? Quelles conséquences emporte-t-elle sur leur organisation administrative, la nature de leur personnel et les relations de ce dernier avec les internés ? La diversité des structures existant sur le sol algérien implique de circonscrire avec précision la notion de "camp d'internement", avant de comparer, de part et d'autre de la Méditerranée, les administrations, le profil des personnels, ainsi que le cadre général déterminant leurs rapports avec les détenus: celui d'une guerre de décolonisation.

\section{Camps d'Algérie, camps de métropole : des réalités complexes à circonscrire et à comparer}

L'usage du seul mot «camp ", pour l'Algérie en guerre, renvoie spontanément aux camps de regroupement, avec lesquels les camps d'internement sont souvent confondus ${ }^{5}$. Ils sont pourtant de nature très différente. Créés à l'initiative de l'armée, les camps de regroupement rassemblaient des familles entières, femmes et enfants inclus, expulsées de leurs zones d'habitat dispersé, où elles pouvaient héberger, ravitailler et renseigner les combattants de l'ALN. Le camp était ici un espace sous étroit contrôle militaire où, en l'absence de toute infra-

5. B. Stora, par exemple, parle du "regroupement des populations » dans des «camps d'hébergement » alors que ces derniers étaient des camps d'internement (Histoire de la guerre d'Algérie, Paris, La Découverte, 2002, p. 15). 
structure économique et sanitaire, les Algériens étaient voués à une misère extrême, dont la surmortalité infantile reste l'indicateur le plus marquant ${ }^{6}$.

Au camp d'internement, en revanche, étaient détenus des individus arrêtés par la police ou par l'armée ; de fait, les internés étaient pratiquement tous des hommes, en dehors du camp de Tefeschoun, dont une partie était réservée aux femmes. Certains camps étaient installés dans des locaux se prêtant à la détention collective, comme un ancien fort, une caserne désaffectée, voire une colonie de vacances dans le meilleur des cas, celui du camp de Lodi ; d'autres ont été rapidement aménagés, dans l'urgence de leur création, avec des baraquements en bois ou des tentes plantées en plein désert. Les conditions d'existence y ont été extrêmement variables, d'un camp à l'autre et d'un moment à un autre, sans jamais susciter, cependant, la même polémique que les camps de regroupement, dont la révélation a fait scandale au printemps $1959^{7}$.

La terminologie administrative, recourant à des euphémismes évitant le mot « camp », concourt aussi au manque de visibilité des camps d'internement. Les documents parlent de "centres de détention administrative » (CDA) ou de «centres d'assignation à résidence surveillée »(CARS). Ces appellations définissent le camp d'internement d'un point de vue juridique : il est le lieu d'une détention non judiciaire, couverte par un arrêté d'assignation à résidence. La loi d'état d'urgence, votée le 3 avril 1955, a en effet autorisé le gouverneur général de l'Algérie et, par délégation, les préfets, à signer de tels arrêtés, à l'encontre de toute personne «dont l'activité s'avère dangereuse pour la sécurité et l'ordre publics $^{8} »$. À cette date, l'internement est conçu comme une mesure préventive de sauvegarde de l'ordre public : il permet de soustraire à un FLN encore peu structuré et à la recherche de troupes, une bonne partie de ceux qui sont susceptibles de le rallier. C'est ainsi que les cadres nationalistes fichés par la police sont arrêtés et internés. Par la suite, après l'abrogation de l'état d'urgence, les décrets des pouvoirs spéciaux, au printemps 1956, ont reconduit l'assignation à résidence, en y ajoutant la possibilité d'une délégation à l'armée ; ils déclenchent l'engrenage conduisant à un usage militaire de la détention dans un camp. Ces textes restèrent le fondement légal de l'internement en Algérie, jusqu'à la fin du conflit.

Reposant sur un critère juridique, cependant, la définition du camp d'internement comme le lieu d'une détention administrative amalgame des réalités très dissemblables. En Algérie, il a existé trois types de camps, classés en fonction de la date de leur création, leur gestionnaire et leur usage : les «centres

6. Cornaton (M.), Les camps de regroupement de la guerre d'Algérie, Paris, L'Harmattan, 1998.

7. À partir d'un rapport de Michel Rocard, alors stagiaire de l'ENA, et divulgué à son insu : Rocard (M.), Rapport sur les camps de regroupement, Paris, Mille et une nuits, 2003.

8. Article 6 de la loi du 3 avril 1955, créant l'état d'urgence. 
d'hébergement » $(\mathrm{CH})$, créés dès 1955 et gérés par les préfectures, où les internés restaient une durée indéterminée, parfois des mois voire des années; les « centres de triage et de transit » (CTT), légalisés en 1957 et gérés par les militaires, où ils étaient censés détenir les individus qu'ils avaient arrêtés; les «centres militaires d'internés » (CMI), ouverts en 1958, également sous la responsabilité de l'armée, réservés à des combattants faits prisonniers que le commandement jugeait opportun de garder sous son contrôle.

Les CTT et les CMI relèvent d'une logique propre à l'armée. Celle-ci considérait l'assignation à résidence comme le moyen de détenir des individus, en toute légalité, au service d'un objectif militaire. Les internés détenus dans les CTT, identifiés comme des lieux possibles de pratique de la torture, étaient soumis à des interrogatoires, et ceux des CMI à une "rééducation ", pour reprendre le vocabulaire en usage à l'époque, considérée comme susceptible de permettre leur enrôlement dans les forces françaises. Servant à la recherche du renseignement ou au retournement de prisonniers, les CTT et les CMI étaient intégrés au dispositif de la lutte contre l'ennemi. Investis d'une telle fonction, les camps gérés par les militaires se distinguent des "centres d'hébergement", où la détention avait pour seul objectif déclaré la mise à l'écart d'individus considérés comme dangereux pour «la sécurité et l'ordre publics ${ }^{9}$ ».

C'était aussi le cas des camps de métropole, qui, entièrement gérés par les autorités civiles, s'apparentent aux seuls « centres d'hébergement » d'Algérie. Tous territoires confondus, les camps se répartissent alors en deux catégories : ceux gérés par les militaires, où ces derniers continuent à faire la guerre, uniquement sur le territoire algérien ; ceux gérés par les civils, sans autre but théorique que la détention de "suspects», en Algérie et en métropole. Une étude comparative entre les deux territoires ne peut donc prendre en compte que cette seconde catégorie.

Les structures métropolitaines sont restées moins étoffées que celles d'Algérie, qui a compté une dizaine de "centres d'hébergement ", de tailles très variables. En avril 1958, par exemple, ils comptaient de 118 internés, à Lodi, le plus petit camp de tous, à environ 1400 dans les deux plus grands, Bossuet et Tefeschoun ${ }^{10}$. Le nombre total d'internés atteint son maximum au printemps 1959, en dépassant les 10000 , avant d'entamer une diminution jusqu'à la fin du conflit ${ }^{11}$. En métropole, seuls quatre camps ont fonctionné, avec une capacité totale de 6000 internés environ ${ }^{12}$. Les deux premiers

9. Ibid.

10. Liste des «centres d'hébergement ", avec leurs effectifs, 2 avril 1958, Service historique de l'armée de terre (SHAT), $1 \mathrm{H} 2573 / 1$.

11. Courbe des effectifs conservée au SHAT, $1 \mathrm{H}$ 1100/1.

12. Liste des camps avec leur date de création et leur capacité, dressée à la fin de la guerre, Centre des archives de Fontainebleau, (CAC), 770119 article 14 (consultation sous dérogation). 
camps, à Mourmelon et Saint-Maurice l'Ardoise, ont été ouverts en 1957, après la première loi autorisant l'assignation à résidence en métropole : votée le 26 juillet 1957, elle posait la condition d'une condamnation judiciaire préalable, limitant ainsi le recours à l'internement. Ce n'est que le 7 octobre 1958 qu'une ordonnance a levé cette restriction: l'assignation à résidence est désormais possible pour toutes les «personnes dangereuses pour la sécurité publique en raison de l'aide matérielle, directe ou indirecte, qu'elles apporteraient aux rebelles des départements algériens ». Cette formulation vague, comme dans les textes qui concernent l'Algérie, élargit considérablement le champ d'application de l'assignation à résidence. Les camps de Thol et du Larzac ont alors été aménagés, pour répondre à la hausse prévisible des effectifs qui s'ensuivrait.

Le nombre de ces camps, ainsi que celui des internés, cependant, est sans commune mesure avec les «six cent mille internés dans quelque deux cents camps $^{13} »$ de la période 1938-1946. Outre que l'internement n'est qu'une facette parmi d'autres de la répression des nationalistes algériens, le contexte de guerre incite les autorités françaises à limiter leur investissement dans des structures de ce type : que les gouvernements soient convaincus de leur capacité à étouffer l'insurrection et garder l'Algérie française, ou au contraire qu'ils s'engagent dans des négociations conduisant à l'indépendance, ils espèrent mettre un terme au conflit. Certes, le contexte de croissance ou de décroissance de l'affrontement avec l'adversaire influence la courbe des effectifs d'internés, orientée à la hausse jusqu'au printemps 1959 avant de connaître une diminution ; mais il n'empêche pas les autorités de penser la guerre, constamment, comme une conjoncture provisoire. Et les camps sont gérés dans cet esprit, avec pragmatisme ${ }^{14}$.

\section{De l'échelon central au camp : une gestion pragmatique}

À l'échelon central, l'organisation de l'administration diverge totalement entre les deux territoires. En métropole, où le ministère de l'Intérieur a la maîtrise de la pratique « internementale », plusieurs services ou bureaux de l'administration centrale sont impliqués. Les décisions d'assignation à résidence relèvent de la compétence du Bureau des assignations à résidence, rattaché à la Direction générale de la Sûreté nationale. Chargé de l'encadrement de la main-

13. Peschanski (D.), La France des camps, op. cit., p. 15.

14. Le caractère provisoire des camps est d'ailleurs l'un des critères de distinction des différents types de camps ayant existé au XXe siècle : cf. Wieviorka (A.), "L'expression "camp de concentration" au XXe siècle ", Vingtième siècle. Revue d'histoire, 54, 1997. 
d'œuvre maghrébine par l'action sociale, le Service des affaires musulmanes et de l'action sociale (SAMAS) intervient également, à l'échelon central et localement, par ses délégués régionaux et départementaux, qui poursuivaient leur tâche à l'intérieur des camps où ils étaient affectés.

D'autres ministères que celui de l'Intérieur ont aussi été sollicités ponctuellement. Le ministère du Travail et celui de l'Éducation nationale ont été associés à des réflexions sur la mise en place d'une formation professionnelle des internés, envisagée pour contrarier l'influence du FLN. Les pouvoirs publics considéraient en effet l'instabilité économique et sociale, fragilisant l'individu, comme un facteur favorable à l'implantation du nationalisme et estimaient vain de libérer des internés sans leur avoir offert, préalablement, des possibilités d'insertion sociale. À plus court terme, une telle formation répondait aux doléances exprimées dans les rapports envoyés au ministère de l'Intérieur : leurs auteurs déploraient l'« oisiveté » des internés, qui laissait le champ libre au FLN, dont les militants s'organisaient dans les camps ${ }^{15}$.

En Algérie, l'administration centrale a été confiée à un service créé par un arrêté du gouverneur général, Jacques Soustelle, le 4 juillet 1955 : le Service central des centres d'hébergement (SCCH). Rattaché au Gouvernement général de l'Algérie, il était dirigé par un préfet, assisté de six personnes : un commissaire principal, un fonctionnaire détaché de la Sûreté générale et quatre personnels administratifs pour le secrétariat, le courrier ${ }^{16} \ldots$ Sa création fait suite au refus de la direction du Travail du Gouvernement général, qui avait pris en charge l'administration des camps pendant la seconde guerre mondiale, de s'y engager de nouveau en 1955, quand sont ouverts les premiers "centres d'hébergement ${ }^{17}$ ». Si la seconde guerre mondiale est une expérience connue, présente dans les réflexions et les débats sur l'internement, y compris à l'Assemblée nationale, lorsque les députés discutent des lois d'exception, elle constitue avant tout un précédent à ne pas reproduire.

Il n'existe donc pas d'administration pérenne et commune aux deux territoires, chargée de l'organisation et de l'administration des camps. Les pouvoirs publics mobilisent des services existant en métropole et créent un service spécifique en Algérie. Cette configuration a une conséquence sur le recrutement du personnel des camps : il échappe à une ligne directrice explicitement définie. Si

15. Pour la métropole, le corpus de base de cet article comprend les rapports envoyés par les délégués locaux du SAMAS à leur administration centrale et conservés au Centre historique des archives nationales (CHAN), sous la cote F1A 5133 (consultation sous dérogation).

16. Arrêté de création du SCCH, 4 juillet 1955, et série de décisions y affectant du personnel, 8 juillet 1955, Centre des archives d'outre-mer (CAOM), CGA 11CAB/38 (consultation sous dérogation).

17. Compte rendu de la réunion du 29 juin 1955, organisée au Gouvernement général et relative à l'état d'urgence, CAOM, CGA 11CAB/38 (consultation sous dérogation). 
une administration spécialisée, pérenne, avait existé, au contraire, elle aurait pu élaborer et appliquer, au fur et à mesure de son expérience, des principes repérables a posteriori par l'historien. Ici, la question du profil de ce personnel se heurte au risque d'une reconstruction rationnelle là où des logiques variables, aléatoires et fragiles, ont été à l'œuvre. Le pragmatisme d'une gestion à l'économie, par ailleurs, s'oppose à l'établissement d'un profil.

Les pouvoirs publics ont en effet été guidés, avant tout, par le souci de limiter leur effort financier. Le personnel du camp est réduit au minimum requis pour son fonctionnement, entre tâches administratives, veille sanitaire et impératifs de sécurité : un directeur, un secrétaire, un gestionnaire, un service médical, une surveillance à l'intérieur et une garde extérieure composent le panel de référence des auteurs de rapports administratifs, qui commencent leur description des camps par une présentation du personnel ${ }^{18}$. Or, ce panel est loin d'être toujours réalisé. Un rapport sur le camp de MourmelonVadenay, en décembre 1957, estime ainsi nécessaire d'embaucher dix personnes, secrétaire, dactylo, magasinier, " hommes à tout faire », infirmier... alors que le directeur utilise l'un des huit surveillants pour faire fonction de secrétaire et qu'aucun représentant du corps médical n'est présent en permanence $^{19}$. En outre, les postes prévus peuvent rester inoccupés, y compris dans un domaine très sensible comme celui de la surveillance intérieure. C'est le cas du camp Paul-Cazelles, en Algérie, dépourvu de surveillants en février 1961, le directeur «s'étant trouvé dans l'impossibilité d'en recruter sur place en raison de l'isolement du lieu ${ }^{20} »$. Les statuts du personnel, enfin, témoignent de la recherche d'une limitation des coûts et des embauches : des militaires à la retraite, des militaires détachés et des contractuels exercent une grande partie de ces fonctions.

Concrètement, en Algérie, le directeur du camp pouvait être un militaire à la retraite, désigné dans les rapports administratifs comme « le colonel commandant le centre », ou un officier de police. En métropole, le directeur, qui n'est pas désigné par un grade, est un civil, comme au Larzac, où ont officié deux commissaires divisionnaires « ayant longtemps servi en AFN (Afrique française du nord $)^{21} »$. Sur les deux territoires, le service médical est généralement confié à des militaires, médecins ou infirmiers, qui peuvent être détachés en permanence dans le camp, intervenir par des consultations régulières ou encore être appelés ponctuellement. Dans le cas où le personnel militaire

18. Outre les rapports du SAMAS pour la métropole, cet article se fonde, pour l'Algérie, sur les rapports d'enquête de la Commission de sauvegarde des droits et libertés individuelles, conservés au CAOM, cotes FM $81 \mathrm{~F} 854$ à 862 (consultation sous dérogation).

19. Rapport du 10/11 décembre 1957, CHAN, F1A 5133.

20. Rapport du 28 février 1961, CAOM, FM $81 \mathrm{~F} 856$.

21. Rapport du 22 juin 1959, CHAN, F1A 5133. 
ne peut assurer les soins médicaux, les autorités font appel à des civils, médecins généralistes, dentistes ou encore pharmaciens, rémunérés en fonction de leurs prestations.

En ce qui concerne la garde extérieure et la surveillance à l'intérieur des camps, enfin, la règle d'un investissement minimal des pouvoirs publics est accentuée par la nécessité de réserver les forces de l'ordre pour des missions plus urgentes, en ces temps de guerre. La garde extérieure revient ainsi aux gendarmes, aux CRS, et aux formations militaires affectées dans le secteur où le camp est installé. En Algérie, quand les opérations offensives mobilisent les troupes régulières, il peut s'agir de forces auxiliaires, comme les Groupes mobiles de protection rurale (GMPR), composées de supplétifs algériens, ou les Unités territoriales (UT), qui recrutaient parmi les Français d'Algérie ${ }^{22}$.

L'emploi de contractuels, recrutés localement pour la surveillance interne, relève de cette même logique d'affectation prioritaire des forces de l'ordre à d'autres tâches. Au Larzac, ce recrutement a puisé dans le vivier des demandeurs d'emploi locaux ${ }^{23}$. À Mourmelon-Vadenay, les huit surveillants et leur chef ne sont présents au camp que 95 heures par semaine, dont 60 heures de travail effectif ${ }^{24}$. L'impossibilité de recruter des surveillants aux alentours du camp de Paul-Cazelles prouve que le recrutement local est aussi pratiqué en Algérie.

Le personnel assure globalement un faible taux d'encadrement des internés à l'intérieur du camp, et ceux-ci participent d'ailleurs à sa gestion en s'occupant de la préparation des repas, en effectuant des travaux de maintenance ou en gérant la cantine, notamment. Ils disposent également de délégués qui, intermédiaires avec l'administration du camp, jouent un rôle à double tranchant : s'ils servent le dialogue en cas de besoin, ils assurent, eux aussi, de fait, l'encadrement de la collectivité internée. Ce sont les militants du FLN qui la structurent, donnant lieu à une dénonciation récurrente, dans les rapports, de l'influence de l'organisation nationaliste dans les camps. Un soin tout particulier est alors apporté au recrutement d'un personnel chargé de la contrecarrer : le «moniteur d'action psychologique» en Algérie et le «conseiller social» en métropole, pendant l'un de l'autre de part et d'autre de la Méditerranée. Leur mission commune n'est rien moins que d'étouffer l'adversaire, en lui soustrayant sa base sociale. Dans l'esprit de ceux qui l'ont conçu, la réussite de ce travail de sape de l'adhésion à la cause de l'adversaire, dépend très largement de la capacité des hommes recrutés à le mettre en œuvre. Au contraire des autres

22. Sur les forces supplétives, cf. Ageron (C.-R.), "Les supplétifs algériens dans l'armée française », Vingtième siècle. Revue d'histoire, 48, 1995.

23. Rapport du 22 juin 1959, CHAN, F1A 5133.

24. Rapport du 10/11 décembre 1957, CHAN, F1A 5133. 
personnels, les autorités ont alors en tête des profils idéaux pour les candidats à "l'action psychologique» ou «l'action éducative et sociale», que l'étude de quelques cas s'y rapportant précisément permet de reconstituer.

\section{Le moniteur d'action psychologique et le conseiller social : deux agents pour une même conception de l'interné}

Les moniteurs d'action psychologique sont des soldats envoyés dans les " centres d'hébergement ", à partir de 1957, à l'initiative du commandement et avec l'accord des autorités civiles. Leur présence exprime l'extension de la conception militaire de l'internement aux «centres d’hébergement». En effet, ces soldats devaient travailler à la "rééducation » politique des internés, avant leur libération. Du point de vue des autorités, en effet, alors que la guerre s'enlisait sans perspective de règlement, l'internement de milliers d'Algériens se prolongeait sans offrir d'issue : comment libérer des internés qui l'ont été, précisément, parce que leur «activité s'avèr[ait] dangereuse pour la sécurité et l'ordre publics » ? La « rééducation » de l'interné s'apparente ainsi à une neutralisation de l'ennemi potentiel qu'il représente, avant sa remise en liberté.

Le $5^{\mathrm{e}}$ bureau de l'armée, chargé de l'action psychologique, cependant, allait plus loin dans ses projets : lorsqu'elle a été proposée au ministre résidant Robert Lacoste en juillet 1956, cette "rééducation » était censée conduire à la formation de "propagandistes algériens", après une "action de désintoxication des prisonniers bien menée ${ }^{25} »$. Elle s'inspire directement de l'expérience des prisonniers français du Viêt-minh, frappés par l'efficacité de la propagande à laquelle ils ont été eux-mêmes soumis ${ }^{26}$.

Les participants à un stage organisé par le $5^{\mathrm{e}}$ bureau à Alger en avril 1957, constituent un échantillon significatif des critères retenus par le commandement pour définir le profil du moniteur d'action psychologique. En douze jours, les stagiaires devaient recevoir une formation générale se déclinant du général au particulier. À des préliminaires sur l'Algérie succédaient une initiation aux principes de "l'action psychologique», une présentation des "centres d'hébergement », puis un exposé des trois phases théoriques de "rééducation »: "reprise en main de l'hébergé », travail sur la « conscience collective », " préparation à la

25. Fiche de renseignements rédigée par le lieutenant-colonel Bruge, lui-même ancien prisonnier du Viêtminh, transmise à Robert Lacoste le 2 juillet 1956, SHAT, 1 H 2573/1.

26. Pour plus de précisions sur cette « rééducation », cf. Villatoux (M.-C.), « Traitement psychologique, endoctrinement, contre-endoctrinement en guerre d'Algérie : le cas des camps de détention ", Guerres mondiales et conflits contemporains, 208, 2002 ainsi que Thénault (S.), «D’Indochine en Algérie : la rééducation des prisonniers dans les camps de détention », La guerre d'Algérie au miroir des décolonisations, Paris, SFHOM, 2002. 
libération ${ }^{27} »$. Dans une fiche relative au déroulement du stage, le commandement évalue les participants. Il expose ainsi ses attentes et dessine, en négatif, les représentations de l'interné qui les sous-tendent.

Est qualifié de "bon», le choix de stagiaires ayant fait des "études secondaires ", leur niveau intellectuel devant garantir une capacité de raisonnement et d'argumentation ${ }^{28}$. Les compétences éducatives et pédagogiques sont aussi appréciées : les professionnels de l'enseignement, trois professeurs et quatre instituteurs, représentent la majorité des stagiaires, juste devant les séminaristes, au nombre de cinq. La présentation d'un stagiaire comme un "moniteur d'enfants » révèle en creux la conception de l'interné véhiculée par les promoteurs de la " rééducation » : cette dernière l'infantilise ; l'interné, pris en charge, est considéré comme un mineur sur le plan politique, qu'il faut orienter dans ses choix. La présence de cinq séminaristes pourrait ainsi s'expliquer : seraient-ils à l'aise dans ce rôle, eux qui se destinent à une mission de guide des consciences?

Le choix des stagiaires, cependant, signale le scepticisme du commandement local envers les principes de l'" action psychologique». En effet, «quelques cultivateurs, ouvriers, chauffeurs » et même " un illettré » ont été désignés par leurs supérieurs pour suivre le stage ; une mention manuscrite en marge du document préconise d'ailleurs de sanctionner «le chef de corps » ayant envoyé ce dernier. En outre, aucun «casting politique », pour reprendre les termes de la fiche relative au déroulement du stage, n'ayant été effectué, il est prescrit aux formateurs de « déceler les "progressistes" s'il y en a ». Enfin, aucun participant n'avait été informé du but du stage.

Les réticences du commandement local à investir dans une tâche à laquelle, au fond, il ne croit guère, contribuent donc à mettre en échec les projets du $5^{\mathrm{e}}$ bureau. Mais les tenants de "l'action psychologique" se heurtent aussi au caractère provisoire de ce personnel, en situation d'incorporation sous les drapeaux. Deux des stagiaires d'avril 1957 étaient ainsi libérables trois mois plus tard. Enfin, le personnel civil faisant défaut dans les " centres d'hébergement ", les moniteurs d'action psychologique y ont été affectés à des postes de secrétaires, de vaguemestres ou de surveillance des internés. Les directeurs de camps y voyaient ainsi un personnel supplémentaire comblant les lacunes du recrutement et les chargeaient, notamment, de l'appel des internés, de la gestion de leurs dossiers ou de donner un avis sur leur libération ${ }^{29}$.

27. Tableau présentant le programme du stage, du 8 au 20 avril 1957, SHAT, 1H 2573/1.

28. Fiche du 11 avril 1957, relative au déroulement du stage, SHAT, 1H 2573/1.

29. Plusieurs documents conservés au SHAT, sous la cote $1 \mathrm{H} 2573 / 1$, expriment les doléances du $5^{\mathrm{e}}$ bureau à ce sujet. 
La présence de ces soldats dans les camps d'internement civils signale la spécificité de la structure internementale en Algérie, siège de la guerre, où l'armée se déploie et accroît ses pouvoirs. La présence de "moniteurs d'action psychologique » et leur détournement par les autorités civiles qui les désignent pour d'autres tâches, est emblématique du rapport entre autorités civiles et autorités militaires. Ces dernières tentent une ingérence dans les «centres d'hébergement », qu'elles dotent d'un objectif militaire, pour lesquelles elles détachent du personnel, après l'avoir formé. Les autorités civiles leur opposent une force d'inertie contrariant, in fine, leurs projets. Les rapports mentionnent alors, par la suite, un officier chargé de l'action psychologique, qui semble être un militaire à la retraite recruté pour prendre en charge la vie collective des internés à l'intérieur du camp, et notamment la discipline ${ }^{30}$.

L' «action psychologique» se présente comme la version militaire d'une conception partagée par les autorités civiles en métropole, sans qu'il y ait eu de concertation et sans que des passerelles aient pour l'instant été établies entre les deux rives de la Méditerranée. En métropole, en effet, les autorités civiles déploient une « action éducative et sociale », confiée à un «conseiller social », qui est recruté en tant que spécialiste du monde colonial. Cette action joue sur une politique d'aide sociale dispensée aux internés, dans plusieurs domaines : maintien des allocations familiales versées à leur famille, maintien de leurs droits éventuels à la Sécurité sociale, assistance aux familles en cas de problème de logement, intervention auprès de l'employeur, tenté de licencier et de remplacer son salarié absent sans motif et pour une durée indéfinie... Cette aide sociale repose sur une analyse du succès du FLN en termes sociaux et non politiques : le mouvement nationaliste prospérerait sur un fond de désœuvrement, d'instabilité et de misère. L'existence d'une nation algérienne est écartée de cette analyse, qui ne l'envisage même pas.

Cette action est confiée au personnel spécialisé du SAMAS, dont les délégués locaux, des administrateurs civils, sont détachés dans les camps, ainsi qu'à un « conseiller social » embauché en dehors du SAMAS. Ce «conseiller social » est recruté, d'abord et avant tout, comme un spécialiste du monde colonial, parmi ceux que les autorités considèrent comme les «intimes des Arabes ${ }^{31}$ ». La priorité est toujours donnée à un connaisseur du Maghreb colonisé, voire un praticien de la domination coloniale. Un vécu, une expérience personnelle, une formation et une carrière professionnelles au Maghreb sont retenus comme critères d'une spécialisation recherchée, ainsi que la maîtrise de l'arabe. En octobre 1959, au Larzac, par exemple, le conseiller social est un ancien sous-officier

30. Cf. plus loin le cas du camp de Bossuet. Il est difficile d'en dire plus dans l'état actuel de la recherche.

31. Abdelfettah (N.), "Science coloniale" et modalités d'encadrement de l'immigration algérienne à Paris », Bulletin de l'IHTP, 83, 2004, p. 116. 
des Affaires indigènes du Maroc. Son adjoint est présenté comme un ancien employé municipal du Constantinois, où il a travaillé douze ans, parlant parfaitement l'arabe. Quant à la femme du conseiller social, qui a vécu au Maroc, elle est employée pour l'accueil des familles venues rendre visite aux internés ${ }^{32}$.

La permanence d'aide sociale peut aussi être sous-traitée à une association, soigneusement choisie. C'est ainsi qu'à Mourmelon-Vadenay, l'« agent social» est recruté par le Comité marno-meusien d'étude et d'action nord-africaine, qui reçoit une subvention du ministère de l'Intérieur pour cette activité3 ${ }^{33}$. En novembre 1958, les autorités interviennent cependant dans le choix de «l'agent social ", fait avec « un soin tout particulier ${ }^{34}$ ». Il repose sur des critères testant particulièrement sa connaissance du monde colonial, qu'il doit réinvestir en métropole : «spécialiste des questions nord-africaines, titulaire du brevet de sociologie musulmane, parlant couramment l'arabe et quelques dialectes. » C'est aussi un ancien capitaine, familier de l'autorité et de la hiérarchie. Le candidat avait d'ailleurs argumenté sa candidature en ces termes : "Je suis né [... en Tunisie », «j'ai étudié l'arabe », « je suis resté treize ans en Algérie en deux périodes» dont dix ans de service dans un "groupe d'aviation de bombardement»; il a également été "officier de sécurité dans l'armée de l'air ${ }^{35}$ ». La familiarité avec l'univers militaire lui conférerait des qualités de discipline, d'autorité, de hiérarchie. L'expérience de la société coloniale et de la guerre est mise en avant et est effectivement déterminante dans le choix de l'agent social.

« Action psychologique » et « aide sociale et éducative » postulent la malléabilité des internés et la possibilité de les détacher du FLN. L'interné ne serait pas un individu doté d'une libre conscience déterminant ses propres choix d'engagement ou de soutien au nationalisme ; mineur politique ou instable désœuvré, il peut - et doit - être guidé, orienté, voire manipulé. L'«action psychologique » et l'«aide sociale et éducative » témoignent d'une conception similaire de l'interné, même si elles mettent en ouvre des approches divergentes, l'une directement politique et relevant d'une version militaire de la guerre, l'autre se déclinant sur un mode socio-économique, propre à des autorités civiles gérant de longue date une main-d'œuvre immigrée sur son sol. Pourtant, les relations du personnel avec les internés se situent sur un autre terrain : organisés, membres d'un camp adverse, ils résistent, voire affrontent les autorités du camp, en métropole et en Algérie, même si leurs marges de manœuvre diffèrent entre les deux territoires.

32. Rapport du 9 octobre 1959, CHAN, F1A 5133.

33. Rapport du 10/11 décembre 1957, CHAN, F1A 5133.

34. Note du SAMAS, datée de novembre 1958, CHAN, F1A 5133.

35. Lettre de candidature adressée au président de l'association et transmise au ministère de l'Intérieur, CHAN, F1A 5133. 


\section{Un contexte extérieur déterminant}

En métropole, les pouvoirs publics tentent de contrôler et limiter les intrusions dans l'espace du camp. C'est ainsi que le ministère de l'Intérieur circonscrit très soigneusement l'intervention d'associations indépendantes, comme le CIMADE (Comité inter-mouvements auprès des évacués) qui sollicite, en novembre 1957, l'autorisation d'" assurer une permanence d'aide morale et sociale $^{36}$ » au camp de Mourmelon-Vadenay. Le ministère est embarrassé par la légitimité de la requête, alors que, précisément, le gouvernement a répondu au scandale public de la torture en affichant une volonté de transparence, par la formation de commissions d'enquêtes; il se dédirait en rejetant la demande du CIMADE concernant un camp métropolitain. Mais le ministère ne peut l'agréer qu'en partie : l'aide sociale doit «demeurer étroitement subordonnée aux autorités responsables du centre ». Admettant pourtant qu'il est difficile de s'opposer à un «concours exceptionnel » du CIMADE, le service du SAMAS l'accepte " sous réserve que son intervention puisse se faire sans contacts directs avec les hébergés et dans des domaines extérieurs au centre (relations avec les familles, remise au travail à la sortie, envoi de jeux au directeur... $)^{37} »$.

L'intervention d'une association indépendante contrarierait la volonté des autorités d'instrumenter l'aide sociale en la confiant au personnel du SAMAS ou à une association sous contrôle. Le choix d'une telle association, qui accepte de se prêter au jeu d'une aide sociale instrumentée à des fins politiques, permet aux autorités d'opposer une fin de non-recevoir aux demandes du CIMADE, sans s'exposer à une dénonciation publique de l'absence d'aide sociale dans les $\mathrm{camps}^{38}$. La réponse des autorités gère alors une double contrainte : s'il est peu opportun de laisser intervenir des associations indépendantes, il est aussi politiquement risqué de les éconduire. La concession d'une aide limitée leur permet d'ajuster au mieux la gestion de cette difficulté.

Le fait même que les autorités acceptent une aide limitée témoigne de la pression dans laquelle sont gérés les camps de métropole. L'existence d'un espace public, où les scandales sont possibles, est caractéristique de ce territoire. Les révélations potentielles créent une pression sur les pouvoirs publics, qui adoptent une attitude prudente. Ils déploient notamment de multiples précautions pour éviter une catastrophe sanitaire, en particulier au Larzac où les risques d'épidémie sont importants, en raison de conditions météorologiques

36. Lettre du secrétaire général du CIMADE au directeur général de la Sûreté nationale, 4 novembre 1957, CHAN, F1A 5133.

37. Avis sur la demande du CIMADE, donné par le SAMAS, 18 janvier 1958, CHAN, F1A 5133.

38. La Croix-Rouge a formulé la même demande pour le Larzac, en juin 1959. CHAN, F1A 5133. 
extrêmement rudes l'hiver, et du nombre élevé d’internés qui y est détenu. Les rapports de l'administration témoignent d'une inquiétude constante à ce sujet.

Cette configuration permet aux internés de tenter de contrarier l'encadrement voulu par les autorités françaises, ainsi que la prise en charge soigneusement réfléchie qui leur est imposée. En juin 1959, par exemple, les internés du Larzac font la grève des soins médicaux, pour protester contre une mesure du directeur du camp : "Il nous semble qu'on frise en permanence l'accident extrêmement grave " écrit alors le délégué du SAMAS, « ce qui peut mener tout droit une certaine presse à faire éclater ce qu'elle titrera "le scandale du Larzac"39. " Puis, en septembre 1959, après le discours du général de Gaulle sur l'autodétermination, il note aussi « un certain durcissement » chez les assignés qu'il nomme «nos adversaires ${ }^{40} »$. Les internés, membres de la partie belligérante opposée à la France, y puisent une force et une capacité de réaction. Appartenant à un camp adverse organisé, avec lequel ils sont en communication, ils peuvent résister à la coercition de l'internement.

Ils ne s'en tiennent pas, en outre, à la défensive. Grâce à l'organisation du FLN à l'intérieur des camps, ils peuvent s'associer à l'action politique menée à l'extérieur. C'est ainsi que le 18 octobre 1961, les internés boycottent la permanence de «l'agent social» de l'association intervenant à Mourmelon-Vadenay $^{41}$. S'ils ne s'en expliquent pas, les autorités formulent l'hypothèse qu'ils ont souhaité s'associer aux manifestations parisiennes de la veille ${ }^{42}$. Puis, en novembre 1961, alors que le FLN lance en métropole une grève de la faim dans les prisons pour obtenir le régime politique, les internés de ce même camp s'y associent pendant cinq jours, par solidarité : « Ils continuent à ne pas douter de la conclusion rapide des pourparlers réglant une fois pour toutes l'affaire d'Algérie ${ }^{43} »$. Ainsi, les informations et les consignes circulent entre l'extérieur et l'intérieur du camp. Les deux espaces sont en interaction. Les internés se battent en lien avec l'extérieur, ils relaient le combat conduit par le FLN en dehors du camp et ils réagissent à l'évolution générale du conflit.

39. Rapport du 22 juin 1959, CHAN, F1A 5133.

40. Rapport du 9 octobre 1959, CHAN, F1A 5133.

41. Rapport du 23 octobre 1961, CHAN, F1A 5133.

42. On pourrait estimer paradoxal le fait que les autorités craignent un scandale public à propos de leur gestion des camps, alors qu'elles n'en tiennent pas compte le soir du 17 octobre 1961, lorsque la manifestation des Algériens est brutalement réprimée en plein cœur de Paris. Cependant, outre que l'initiative de la base policière a joué son rôle dans cette répression, celle-ci peut aussi être assumée par les autorités de l'État comme une réponse à une décision prise par l'adversaire. Les défenseurs de l'action des forces de police ce soir-là, ont développé cet argument en faisant porter la responsabilité de la répression au FLN, qui, connaissant l'état d'esprit des policiers parisiens exaspérés par les attentats, aurait joué avec le feu. Une telle argumentation n'aurait pas pu être tenue en cas d'épidémie ravageuse dans les camps, ni même dans le cas d'une répression sur des internés passifs.

43. Rapport du 20 novembre 1961, CHAN F1A 5133. 
En Algérie, siège de la guerre, le camp ne fonctionne pas non plus comme un espace coupé de l'extérieur. Les internés y sont fragilisés et exposés à des traitements dégradants pouvant aller jusqu'aux sévices ou à la mort ${ }^{44}$. Les insultes proférées par les gardes extérieurs ou surveillants intérieurs sont souvent mentionnées par les enquêteurs, en particulier lorsque les Unités territoriales (UT) y participent. Ces formations supplétives composées d'Européens ont en effet attiré les activistes de l'Algérie française et sont devenues des foyers de l'agitation ultra. Un conseiller de l'Union française écrit même au Premier ministre pour dénoncer le passé des deux assassins de l'avocat libéral $\mathrm{M}^{\mathrm{e}}$ Popie : tous deux membres de l'OAS, ils ont appartenu à une UT affectée à la garde du camp de Berrouaghia ${ }^{45}$.

La garde des camps, en Algérie, se heurte ainsi à un problème spécifique : le recours à des forces supplétives conduit à l'emploi d'activistes investis, par leurs fonctions de gardiens ou de surveillants, d'un pouvoir légitime sur leurs adversaires directs que sont les internés nationalistes. En février 1961, les membres de la Commission de sauvegarde consacrent un paragraphe de leur rapport à ce problème, après avoir été eux-mêmes pris à partie par ces partisans de l'Algérie française qui rejettent la politique du général de Gaulle : les gardiens du camp de Tefeschoun ont entamé le chant des Africains et crié "Algérie française » à leur passage. "À Douera, on pouvait lire au-dessus du poste de garde des inscriptions telles que "Vive Lagaillarde" et "Algérie française" ", relatent-ils encore ${ }^{46}$.

En Algérie, également, la discipline instaurée par les directeurs à l'intérieur des camps, libres de toute initiative en la matière, révèle la tension qui règle les rapports entre le personnel et les internés. En février 1961, encore, les enquêteurs, comparant trois camps qu'ils viennent de visiter, font état d'un traitement des internés variable d'un camp à l'autre, certes, mais qui s'inspire éventuellement de la discipline miliaire : le salut aux couleurs est obligatoire à Tefeschoun, facultatif à Paul-Cazelles, inexistant à Douera. Après une rencontre avec le directeur du $\mathrm{SCCH}$, à qui le problème a été posé, les enquêteurs rapportent que les sanctions ont été "déterminées avec précision » : la suppression du courrier a disparu du panel des punitions éventuelles et « la peine de cellule qui, selon les camps, excédait parfois plusieurs mois, ne pourra à l'avenir dépasser quinze jours $^{47} »$. Leur démarche prouve a contrario l'absence de consignes encadrant strictement les pouvoirs du directeur du camp et de son personnel, à un an de la fin de la guerre, alors que les «centres d'hébergement » comptaient déjà six ans

44. Sur le traitement des internés en métropole et une comparaison avec celui des internés en Algérie, cf. l'article de M. Bernardot dans ce même numéro : « Être interné au Larzac. La politique d'assignation à résidence surveillée durant la guerre d'Algérie (1958-1962)».

45. Lettre de Jean Scelles au Premier ministre, arrivée le 18 mars 1961, CAOM, FM 81F 856.

46. Rapport du 28 février 1961, CAOM, FM $81 \mathrm{~F} 856$.

47. Rapport du 28 février 1961, CAOM, FM 81F 856. 
d'existence. Dans leur rapport du 3 janvier 1962, d'ailleurs, ils s'attardent sur la situation du camp du Maréchal, où le colonel en retraite, directeur du camp, «toujours en tenue », " exige les marques de respect définies par le règlement de l'armée », « supprime les journaux » et «imposerait des corvées ${ }^{48}$ ».

Ces rapports tardifs peuvent suggérer que les partisans de la répression, qui refusent la politique du général de Gaulle, trouvent dans l'humiliation des internés un exutoire à leur frustration de devoir bientôt cesser la lutte contre l'adversaire ${ }^{49}$. Mais, de tels comportements étant aussi décrits dès 1957, ils expriment plutôt une volonté de coercition constante, qui peut, suivant le moment où elle se produit, être en cohérence ou non avec la politique gouvernementale $e^{50}$. Le contrôle des forces répressives agissant en Algérie étant défaillant, il était impossible d'ajuster leurs attitudes à cette politique, lorsque celle-ci a pris la voie de la négociation ; le général de Gaulle, d'ailleurs, ne l'a pas recherché : mettre un terme au conflit était la seule véritable solution pour reprendre en main tous ceux qui, à la faveur de l'aventure algérienne, se sont affranchis du contrôle du pouvoir central ${ }^{51}$.

C'est également dans cette liberté d'action laissée au personnel que naissent toutes les possibilités de mauvais traitements et de morts suspectes d'internés, comme à Bossuet au printemps 1959. Selon la version communiquée par les autorités d'Alger, l'arrivée d'un officier en retraite, affecté au titre de l' " action psychologique », partisan du travail forcé des internés, qu'il frappait et qu'il punissait en leur imposant des corvées supplémentaires, a déclenché l'engrenage d'un affrontement meurtrier. Au mois de mai, les internés ont entamé une grève de la faim pour protester contre le régime des corvées de nettoyage et demander une augmentation de la rémunération perçue pour les travaux effectués à l'intérieur du camp. Obtenant satisfaction de la part du directeur du centre, ils en ont déclenché une seconde en juin pour réclamer une amélioration et une augmentation de leur nourriture. Ils se sont aussi opposés à la gendarmerie, venue enquêter à l'intérieur du camp, qu'ils ont accueillie avec des chants et des slogans. Les interventions des CRS et d'un peloton de gendarmerie, à deux reprises, ont alors fait plusieurs blessés, dont l'un serait mort ensuite. Or, le PV de gendarmerie a camouflé ce décès en suicide; selon une autre version des

48. Rapport du 3 janvier 1962, CAOM, FM 81F 856.

49. C’est aussi le cas des peines capitales, prononcées par les tribunaux militaires jusqu'au 9 mars 1962, alors que la dernière exécution a eu lieu en décembre 1960, le GPRA ayant fait savoir qu'il refuserait de négocier si de nouvelles exécutions avaient lieu. Les membres de ces tribunaux manifestaient ainsi leur désaccord avec la politique suivie à Paris. Cf. Thénault (S.), Une drôle de Justice. Les magistrats dans la guerre d'Algérie, Paris, La Découverte, 2004, p. 280-284.

50. C’est le cas du camp de Paul-Cazelles en 1957. Cf. Martin-Chauffier (L.), « Journal en marge d'une enquête ", Saturne, 16, 1957.

51. Sur le contrôle des camps eux-mêmes par la Commission d'inspection des centres de détention administrative (CICDA), créée à Alger en janvier 1960, cf. Thénault (S.), Une drôle de Justice, op. cit., p. 243-246. 
faits, la mort de l'interné serait même consécutive à des sévices. Un CRS gradé, reconnu coupable de torture, d'ailleurs, a été puni ${ }^{52}$.

Sans être clairement élucidées, les circonstances de cette répression imposée à la résistance des internés révèlent les défaillances dans le contrôle du personnel du camp par les pouvoirs publics, qui réagissent à la visite du camp par la Croix-Rouge internationale, dans le cadre de ses misions ordinaires en Algérie, et pour répondre à la version de ces événements parvenue à la presse métropolitaine. L'officier chargé de l'action psychologique est en effet renvoyé le 30 novembre 1959, quinze jours après la venue de la Croix-Rouge, et les deux rapports d'enquête existant font suite à une double dénonciation : l'une par lettre anonyme parvenue à la Commission de sauvegarde en août 1959, donnant lieu à un rapport du président de la Commission de sauvegarde en janvier 1960 ; l'autre publiée par les Temps Modernes en octobre 1959, qui incite le garde des Sceaux, Edmond Michelet, à demander un rapport au Secrétariat général pour les Affaires algériennes, rendu en mars $1960^{53}$. La chaîne de mobilisation nécessaire à la connaissance de l'affaire, de l'intérieur du camp où les événements ont eu lieu jusqu'à Paris, prend ainsi près d'un an. Elle n'offre pas aux internés d'Algérie une protection suffisante pour leur conférer une marge de manœuvre identique à celle dont disposent les internés métropolitains. Le cadre général des relations entre personnel et internés est ainsi déterminé par le hors champ du camp.

À l'intérieur des camps, comme protagonistes ennemis d'une guerre d'indépendance, personnel et internés inscrivent leurs relations dans un double rapport: un rapport colonial, hérité de la longue durée, matrice de stéréotypes appliqués aux internés, et un rapport de forces, construit collectivement par les Algériens contre cette domination, source de tensions à l'intérieur du camp. Les internés sont traités à la fois comme des adversaires et comme des sujets coloniaux, par la « rééducation » confiée à des soldats désignés «moniteurs d'action psychologique " en Algérie, et par l'action dite "éducative et sociale » qui emprunte les canaux d'une "ethnographie policière de l'immigré nord-

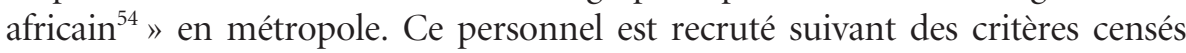
garantir sa compétence dans la prise en charge de cette collectivité, que les représentations coloniales enferment dans la caricature d'une masse manœuvrable.

52. Version des faits d’après le rapport du Délégué général du gouvernement en Algérie, reprise par le président de la Commission de sauvegarde à l'intention du Premier ministre, 20 janvier 1960, CAOM, FM 81F 858.

53. Dossier "camp de Bossuet, incidents de juin 1959 et correspondance avec le garde des Sceaux", CAOM, FM $81 \mathrm{~F} 85$.

54. Abdelfettah (N.), "Science coloniale" et modalités d'encadrement de l'immigration algérienne à Paris », art. cité, p. 118. Plusieurs articles de ce dossier "Répression, contrôle et encadrement dans le monde colonial au XXe siècle » se rapportent à cette question. 
Contrariant et combattant ces efforts des autorités françaises, cependant, les internés, organisés par le FLN, puisent dans le contexte extérieur les ressources d'une résistance variable d'un territoire à l'autre : si, en métropole, ils disposent d'une marge de manœuvre leur permettant de développer des modes d'action spécifiques, en Algérie, en revanche, la tension règle leurs rapports avec le personnel et l'opposition peut conduire à un affrontement, dans lequel ils risquent leur vie. En outre, la chronologie politique, allant de l'enlisement dans la guerre à son règlement par des négociations, marque plus les relations entre internés et personnel en métropole qu'en Algérie, où les forces répressives rechignent à suivre la ligne du pouvoir parisien à partir du moment où ce dernier discute avec l'adversaire. Et ce n'est pas le moindre des paradoxes que de voir, sur ce territoire, l'affrontement se perpétuer, alors que des négociations avec le GPRA (Gouvernement provisoire de la République algérienne) sont en cours.

Sylvie THÉnAuLt est chargée de recherche à I'Institut d'histoire du temps présent (IHTP). Elle est spécialiste des questions de répression légale des nationalistes algériens, thenault@ihtp.cnrs.fr
Parmi ses publications récentes: Une drôle de Justice. Les magistrats dans la guerre d'Algérie, La Découverte, coll. "poches sciences humaines", 2004 (rééd.) ; "Assignation à résidence et justice en Algérie ", Le Genre Humain, 32, 1997. 